\title{
A comparative study of the prevalence of hyperkalemia with the use of angiotensin- converting enzyme inhibitors versus angiotensin receptor blockers
}

This article was published in the following Dove Press journal:

Pathology and Laboratory Medicine International

9 July 2009

Number of times this article has been viewed

\author{
Seyed Ali Sadjadi' \\ James I McMillan' \\ Navin Jaipaul' \\ Patricia Blakely' \\ Su Su Hline ${ }^{2}$ \\ 'Section of Nephrology (I I IN), \\ Jerry L Pettis Memorial Veterans \\ Medical Center, Loma Linda, CA, \\ USA; ${ }^{2}$ Divison of Nephrology, Loma \\ Linda University Medical Center, \\ Loma Linda, CA, USA
}

Background and objectives: Angiotensin-converting enzyme inhibitors (ACEI) and angiotensin receptor blockers (ARB) are increasingly used in a variety of settings including heart failure, renal failure, arterial hypertension, and diabetic nephropathy. The objective of this study was to investigate the prevalence of hyperkalemia with ACEI and ARB use, in a population of the United States veterans.

Design, settings, material, and measurements: Retrospective observational cohort study of 1163 patients on ACEIs and 1168 patients on ARBs in a single Veterans Affairs Medical Center. Electronic medical records were reviewed over a 12-month period with data collected on various demographic, laboratory, comorbidity, and medication related variables.

Results: Hyperkalemia ( $>5 \mathrm{mEq} / \mathrm{L}$ ) was observed in $20.4 \%$ of patients on ACEIs and $31.0 \%$ on ARBs. Severe hyperkalemia (6 mEq/L or higher), was observed in $0.8 \%$ of ACEI and $2.8 \%$ of ARB users. In univariate logistic regression analyses, diabetes mellitus; serum glucose, total carbon dioxide content, creatinine, and estimated glomerular filtration rate (GFR) were significantly associated with hyperkalemia. ARB use, when compared to ACEI, was associated with a $42 \%$ increase in odds of hyperkalemia (odds ratio $[\mathrm{OR}]=1.42 ; \mathrm{p}=0.001$ ) in a model including adjustment for GFR and a $56 \%$ increase in odds of hyperkalemia $(\mathrm{OR}=1.56$; $\mathrm{p}<0.001)$ in a model including adjustment for serum creatinine.

Conclusions: Hyperkalemia, associated with the use of ACEIs and ARBs, is usually mild and severe hyperkalemia is rare. Hyperkalemia is more common with ARBs than ACEIs. ARB use, when compared to ACEI use, may significantly and independently be associated with increased odds of hyperkalemia.

Keywords: hyperkalemia, angiotensin-converting enzyme inhibitors, angiotensin receptor blockers

\section{Introduction}

Over the past four decades, many advances have taken place in the management of heart failure, diabetic nephropathy, arterial hypertension, and chronic kidney disease. These advances have included use of angiotensin-converting enzyme inhibitors (ACEIs), ${ }^{1}$ angiotensin receptor blockers (ARBs), and more recently, renin blockers. Use of $\mathrm{ACEIs}^{2}$ and $\mathrm{ARBs}^{3}$ has contributed to better outcomes in heart failure with less need for hospitalization, improved functional class, and decreased mortality. Similarly, ACEIs and ARBs have shown efficacy in decreasing proteinuria, and slowing the progression of diabetic nephropathy. Their use, however, is sometimes complicated
Correspondence: Su Su Hline Loma Linda University Medical Center Section of Nephrology, I I234, Anderson St Rm I539, Loma Linda, CA 92350, USA Tel + I 9095584906

Fax + I 9095580396

Email shline@llu.edu 
by hyperkalemia that may necessitate their discontinuation. It is also estimated that as many as $5 \%$ to $10 \%$ of patients with congestive heart failure (CHF) may not tolerate ACEIs due to hypotension or azotemia, but do better with ARBs. ${ }^{4}$ Some studies, ${ }^{5}$ have shown a reduced prevalence of hyperkalemia with ARBs possibly due to less suppression of aldosterone secretion, ${ }^{6}$ but others have not confirmed this effect. ${ }^{7}$ If confirmed, substituting ARBs for ACEIs becomes a plausible alternative in patients who desperately need the beneficial effects of renin-angiotensin-aldosterone system blockade. Therefore, we decided to examine and compare the prevalence of hyperkalemia in a large veteran population receiving ACEIs and $\mathrm{ARBs}$ to explore factors that increase the risk of hyperkalemia.

\section{Methods}

We investigated the prevalence, magnitude, and independence of the association of ACEI and ARB use with hyperkalemia in a population of the United States veterans after receiving approval from our local institutional review board. The pharmacy service provided a list of several thousand patients receiving these medications. Through randomized selection, 1,163 patients receiving ACEIs and 1,168 patients receiving ARBs were included in the study. Using a computerized patient record system (CPRS), serum potassium over a 12-month period was reviewed, the highest value identified, and concurrent laboratory values recorded. Information was collected on various demographic, comorbid, and laboratory variables including age, body mass index (BMI), presence or absence of ICD-9-CM classified heart failure (CHF), diabetes mellitus (DM), arterial hypertension (HTN), chronic kidney disease (CKD), serum sodium, potassium, chloride, total carbon dioxide content $\left(\mathrm{CO}_{2}\right)$, blood urea nitrogen (BUN), serum creatinine $(\mathrm{Cr})$, and GFR estimated by a four-variable modification of diet in renal disease formula (MDRD). Information was also collected on medication usage: ACEI type and dose, ARB type and dose, and concomitant nonsteroidal anti-inflammatory drug (NSAID), diuretic, and potassium supplement use. Patients who were on ACEIs or ARBs with missing clinical and laboratory variables were excluded. This accounted for $11 \%$ of screened patients. Hyperkalemia was defined as serum potassium level higher than $5 \mathrm{mEq} / \mathrm{L}$ that was observed on at least one occasion during the 12-month period preceding the analysis. Comparison of groups was done by Student's $t$-test for normally distributed continuous variables and Pearson's chi-squared test for categorical variables. A p-value less than 0.05 was considered significant. Univariate logistic regression was used to determine the association of each variable of interest with hyperkalemia. For each variable significantly associated with hyperkalemia, bivariate logistic regression was performed to determine which of these factors resulted in a greater than $10 \%$ change in the odds of hyperkalemia for ARB use when compared to ACEI use. To achieve optimal model stability, these factors were then included in a multivariate logistic regression analysis to determine the adjusted odds of hyperkalemia for ARB use in reference to ACEI use.

\section{Results}

\section{Comparison of ACEI and ARB users}

Table 1 details demographics, associated medical conditions, simultaneous medication use, and CKD stage of the two groups. There were no significant differences between the two groups with regard to age, gender, BMI, diabetes mellitus, or hypertension; but CHF was more common in ARB users (17.1 vs 9.5\%). Serum creatinine (1.47 \pm 1.03 vs $1.25 \pm 0.64 \mathrm{mg} / \mathrm{dL} ; \mathrm{p}<0.001)$ was significantly higher and

Table I Patient demographics, associated conditions, and medication use

\begin{tabular}{|c|c|c|c|}
\hline & ACEI & ARB & P Value \\
\hline Number & 1163 & 1168 & \\
\hline Mean age, years & $67.5 \pm 10.9$ & $67.2 \pm 10.7$ & 0.439 \\
\hline $\mathrm{BMI}, \mathrm{kg} / \mathrm{m}^{2}$ & $30.7 \pm 6.9$ & $31.3 \pm 7.3$ & 0.025 \\
\hline Diabetes mellitus (\%) & 51.5 & 52.1 & 0.649 \\
\hline Hypertension (\%) & 91.7 & 92.9 & 0.326 \\
\hline Heart failure (\%) & 9.5 & 17.1 & 0.001 \\
\hline Creatinine $\mathrm{mg} / \mathrm{dL}$ & $1.25 \pm 0.64$ & $1.47 \pm 1.03$ & $<0.001$ \\
\hline eGFR ml/min/l.73 m² & $69.0 \pm 21.4$ & $60.9 \pm 20.7$ & $<0.00 \mathrm{I}$ \\
\hline Glucose mg/dL & $130.9 \pm 57.8$ & $141.0 \pm 76.4$ & 0.809 \\
\hline Total $\mathrm{CO}_{2} \mathrm{meq} / \mathrm{L}$ & $27.4 \pm 2.9$ & $27.7 \pm 3.9$ & 0.001 \\
\hline Loop diuretics (\%) & 14.7 & 24.4 & $<0.00$ I \\
\hline Thiazides (\%) & 26.7 & 28.4 & 0.379 \\
\hline $\begin{array}{l}\text { Potassium-sparing } \\
\text { diuretics (\%) }\end{array}$ & 6.1 & II.I & $<0.00 \mathrm{I}$ \\
\hline Potassium chloride (\%) & 6 & 13.1 & $<0.00 \mathrm{I}$ \\
\hline NSAIDS (\%) & 17 & 17.5 & 0.784 \\
\hline CKD stage I (\%) & 16.2 & 8.2 & $<0.001$ \\
\hline CKD stage $2(\%)$ & 51.4 & 46.0 & $<0.009$ \\
\hline CKD stage 3 (\%) & 30.1 & 39.9 & $<0.001$ \\
\hline CKD stage 4 (\%) & 1.7 & 4.6 & $<0.001$ \\
\hline CKD stage $5(\%)$ & 0.6 & 1.3 & $<0.089$ \\
\hline
\end{tabular}

Abbreviations: ACEl, angiotensin-converting enzyme inhibitors; $A R B$, angiotensin receptor blockers; BMI, body mass index; CKD, chronic kidney disease; GFR, glomerular filtration rate; NSAIDs, nonsteroidal anti-inflammatory drugs. 
GFR was significantly lower in ARB users (60.0 \pm 20.7 vs $69.0 \pm 21.4 \mathrm{~mL} / \mathrm{min} / 1.73 \mathrm{~m}^{2}$ body surface area [BSA]; $\mathrm{p}=0.001)$. Use of loop diuretics $(24.4 \%$ vs $14.7 \%, \mathrm{p}<0.001)$, potassium-sparing diuretics $(11.1 \%$ vs $6.1 \%$; p $<0.001)$, and potassium supplements (13.1\% vs $6.0 \%$; $<<0.001)$ was significantly higher in ARB compared to ACEI users. When grouped according to CKD stage, the distribution of GFR was significant for more advanced kidney disease among ARB users: $39.9 \%$ vs $30.1 \%$ for stage III, $4.6 \%$ vs $1.7 \%$ for stage IV, and $1.3 \%$ vs $0.6 \%$ for stage $\mathrm{V}$.

Of the 1,136 patients on ACEI, 831 (71.0\%) were on lisinopril, 225 (19.3\%) on benazepril, 84 (7.2\%) on fosinopril, $12(1.0 \%)$ on enalapril, and $11(0.8 \%)$ on captopril. Among ARB users, 1126 (96.4\%) were on irbesartan and 42 (3.5\%) on losartan (ARB use was restricted by hospital formulary). Mean daily dosage of these medications were as follows: lisinopril $29.4 \pm 13.8 \mathrm{mg}$, benazepril $31.2 \pm 12 \mathrm{mg}$, fosinopril $34 \pm 12.1 \mathrm{mg}$, enalapril $9.5 \pm 3.9 \mathrm{mg}$, captopril $68.7 \pm 47.8 \mathrm{mg}$, irbesartan $202.3 \pm 87.4 \mathrm{mg}$, and losartan $66.6 \pm 33.3 \mathrm{mg}$.

\section{Comparison of hyperkalemic ACEI and ARB Users}

Hyperkalemia, serum potassium $>5.0 \mathrm{mEq} / \mathrm{L}$ on at least one occasion and over a one-year period, was observed in $238(20.4 \%)$ of ACEI users vs 361 (31.0\%) of ARB users. Serum potassium, when stratified by the level of hyperkalemia from 5.0 to $5.50,5.6$ to 5.9 , and $6.0 \mathrm{mEq} / \mathrm{L}$ or higher was seen in $16.6 \%, 4.9 \%$, and $0.8 \%$ of ACEI users vs $22.3 \%$, $5.8 \%, 2.8 \%$ of ARB users, respectively. Among diabetics, 143 (24\%) on ACEI therapy and 229 (37.7\%) on ARB therapy were hyperkalemic, with mean GFR of $68.7 \pm 22.9$ in ACEI vs $58.7 \pm 21.3 \mathrm{ml} / \mathrm{min}$ in ARB users. Hyperkalemia was seen in $30.6 \%$ of patients on irbesartan or losartan, $27.2 \%$ on captopril, $21.7 \%$ on fosinopril, $21.5 \%$ on lisinopril, and $16.5 \%$ on benazepril. One hundred seventy five patients were on combined ACEI and ARB. Of these, $48(27.4 \%)$ had a serum potassium $>5.0,14(8.0 \%)$ had a serum potassium $>5.5$, and $4(2.2 \%)$ had a serum potassium of $6 \mathrm{mEq} / \mathrm{L}$ or higher. Table 2 shows comparison of hyperkalemic patients on ACEIs and ARBs. Among hyperkalemic patients, ARB users had significantly higher mean BUN and serum creatinine, but a lower GFR. Concomitant use of potassium sparing diuretics (17.5\% vs $8.0 \%$ ) and nonpotassium-sparing diuretics (48.0\% vs 38.0\%) was significantly higher among hyperkalemic ARB users.

\section{Logistic regression analysis}

Of the demographic-, comorbidity-, laboratory-, and medication-related variables studied, we identified four
Table 2 Comparison of hyperkalemic patients receiving ACEI or ARBs

\begin{tabular}{llll}
\hline & ACEI & ARB & p value \\
\hline & 238 & 361 & \\
Age, years & $68.6 \pm 10.8$ & $68.2 \pm 10.5$ & NS \\
BMI, Kg/m² BSA & $30.3 \pm 6.3$ & $30.4 \pm 5.8$ & NS \\
Glucose mg/dL & $142.3 \pm 79.4$ & $148.7 \pm 81.0$ & NS \\
BUN mg/dL & $23.8 \pm 14.8$ & $30.2 \pm 16.9$ & $<0.001$ \\
Creatinine mg/dL & $1.44 \pm 0.79$ & $1.82 \pm 1.35$ & $<0.001$ \\
CO $_{2}$ content & $26.89 \pm 3.42$ & $26.73 \pm 3.58$ & $\mathrm{NS}$ \\
DM \% & 60.0 & 63.0 & $\mathrm{NS}$ \\
Potassium-sparing diuretic \% & 8.0 & 17.5 & $0.00 \mathrm{I}$ \\
Nonpotassium-sparing & 38.0 & 48.0 & 0.016 \\
diuretic \% & & & \\
eGFR ml/min/l.73 m ${ }^{2}$ BSA & $60.88 \pm 21.2$ & $51.5 \pm 21.2$ & $<0.001$ \\
\hline
\end{tabular}

Abbreviations: $A C E I$, angiotensin-converting enzyme inhibitors; $A R B$, angiotensin receptor blockers; $\mathrm{BMI}$, body mass index; $\mathrm{BSA}$, body surface area; $\mathrm{BUN}$, blood urea nitrogen; DM, diabetes mellitus; GFR, glomerular filtration rate; NS, Not significant.

factors significantly associated with hyperkalemia, which in bivariate logistic regression analysis were found to result in greater than $10 \%$ change in the odds ratio (OR) of hyperkalemia for ARBs in reference to ACEIs. Specifically, these included serum glucose, BUN, Cr, and GFR. Notably, none of the other variables we studied, including diabetes, $\mathrm{CHF}, \mathrm{CO}_{2}$, nonpotassium-sparing and potassium-sparing diuretic use, NSAID use, or potassium supplement use significantly affected the odds of hyperkalemia for ARBs in reference to ACEIs. Blood glucose, BUN, and $\mathrm{Cr}$ were positively correlated, while GFR was negatively correlated with hyperkalemia ( $\mathrm{p}<0.001)$. Due to collinearity between three of these variables, (BUN, Cr, and GFR), we developed two separate multiple logistic regression models to determine the adjusted odds of hyperkalemia for ARBs in reference to ACEIs. In the first model, we included the GFR, and in the second model, we included the serum Cr. In univariate logistic regression analysis, ARB use was associated with a $73 \%$ increase in crude OR for hyperkalemia $(\mathrm{OR}=1.739 ; \mathrm{p}<0.001)$. After adjusting for blood glucose and GFR, there was still an increased risk of hyperkalemia with ARBs $(\mathrm{OR}=1.43 ; \mathrm{p}=0.001)$. Even after adjusting for blood glucose and serum $\mathrm{Cr}$, risk of hyperkalemia with $\mathrm{ARBs}$ $(\mathrm{OR}=1.56 ; \mathrm{p}<0.001)$ compared to ACEIs persisted.

\section{Discussion}

ACEIs, along with beta blockers, ${ }^{8}$ spironolactone, ${ }^{9}$ and eplerenone ${ }^{10}$ have proven themselves to be of great benefit in the management of patients with left ventricular dysfunction. 
Their use has become part of accepted regimens of treatment and a yardstick of quality of care provided to these patients. ${ }^{11}$ ACEI s and ARBs have also been found to be effective in preserving renal function ${ }^{12}$ in patients with diabetes mellitus and also in nondiabetic conditions like polycystic kidney disease, ${ }^{13,14}$ idiopathic nephrotic syndrome and Ig A nephropathy. ${ }^{15,16}$ Combined use of ACEI and ARB has been advocated by some studies ${ }^{17-19}$ but not all to be more effective in preserving renal function. ${ }^{20}$

Every patient with heart failure and diabetes mellitus, even those that are not hypertensive are expected to receive these medications unless there is an untoward reaction to their use, like, angioedema, progressive renal failure, or hyperkalemia.

Hyperkalemia, because of its serious consequences, like cardiac arrhythmia and death, ${ }^{21,22}$ frequently compels physicians to discontinue these medications, especially in those with kidney disease. Opinions vary as to what level of hyperkalemia requires action. Some would advocate a level of $8 \mathrm{mEq} / \mathrm{L}$ or higher ${ }^{23}$ as grounds for hospitalization, while others feel very uneasy leaving a patient with a serum potassium level higher than $5 \mathrm{mEq} / \mathrm{L}$ on these agents. In our study we found that although hyperkalemia (serum potassium $>5 \mathrm{mEq} / \mathrm{L}$ ) can be seen in as many as $30 \%$ of patients, severe hyperkalemia $(>6 \mathrm{mEq} / \mathrm{L})$, is observed only in about $1 \%$ to $3 \%$ of patients. Higher prevalence of hyperkalemia with ARBs (31\% vs 20.4\%) in our study may be due to higher prevalence of CHF in this group, higher use of potassium-sparing diuretics and potassium replacement therapy, and the switching of hyperkalemic patients from ACEI to ARBs due to the widely held belief among physicians that ARBs cause less hyperkalemia than ACEIs. However, even after accounting for all the above mentioned factors, and although the association weakened, ARB use was still associated with a significant increase in odds risk for hyperkalemia.

In our study hyperkalemia was more common, compared to controlled studies, where ARBs were used primarily for the treatment of heart failure or nephropathy in patient populations with normal or near normal kidney function and where hyperkalemia was not the primary study focus (Table 3). Bakris and colleagues ${ }^{6}$ and Preston and colleagues ${ }^{7}$ compared the effect of ACEIs and ARBs on serum potassium concentration. In the former study, there were 35 patients with a mean GFR of $65 \pm 5 \mathrm{~mL} / \mathrm{min}$ and there was less increase in serum potassium with valsartan than with lisinopril (0.12 vs $0.28 \mathrm{mEq} / \mathrm{L})$. In the latter study, 24 diabetic patients who had normal renal function (Cr clearance $109 \mathrm{~mL} / \mathrm{min}$ ), were treated with candesartan and lisinopril. No difference in fractional excretion of potassium or serum potassium concentration was observed. However, in posthoc analyses of RENAAL study, Winklemayer and colleagues ${ }^{24}$ and Appel and colleagues ${ }^{25}$ reported hyperkalemia (value not reported) in $24.3 \%$ of losartan treated patients vs $12.3 \%$ of controls and discontinuation of treatment in $1.3 \%$ of patients because of hyperkalemia. The prevalence rate of hyperkalemia in this randomized controlled study is very similar to the results of our study.

Some of the hyperkalemia observed in our study may be artifactual, related to the technique of blood drawing ${ }^{26}$ and some due to the fact that most of our patients had moderate to advanced renal and heart failure where there is more likelihood of renal dysfunction and hyperkalemia. ${ }^{27,28}$ However, as previously noted, the observed associations between hyperkalemia and ARB use in reference to ACEI use persisted even after adjustment for renal function.

Our study has some limitations. First is the fact that it was retrospective and observational in nature and it can not provide any information on the time frame of development of hyperkalemia after the start of ACEIs or ARBs. Second, it was limited to a veteran population at a single center and may not be generalizable to other populations. Third, there may have been a tendency among practitioners to preferentially prescribe ARBs over ACEIs to patients with a higher disease severity, introducing the possibility for confounding by indication, a type of selection bias. Lastly, causal inference regarding the effect of ARB use in reference to ACEIs use cannot be drawn from this study due to its observational nature. Nevertheless, the validity of our study findings are supported by the detection of other well established associations between other study variables and hyperkalemia, which occurred in the direction and magnitude expected based on previous reports in the literature.

In conclusion, use of ACEIs and ARBs is associated with a high prevalence of hyperkalemia, but more so with ARBs. Most patients can and should benefit from the beneficial effects of these agents, but caution should be exercised especially in those with advanced kidney disease, heart failure, on renal replacement therapy, on potassium sparing diuretics and $\mathrm{KCl}$ replacement therapy. ${ }^{29}$ We agree with Palmer $^{30}$ that ACEIs or ARBs should be discontinued once serum potassium exceeds $5.5 \mathrm{mEq} / \mathrm{L}$, unless it can be controlled with diet, diuretics or sodium polystyrene sulfonate. As it has been recommended by others, we would advise checking of serum potassium level in one week after any change in ACEI or ARB dosing. 


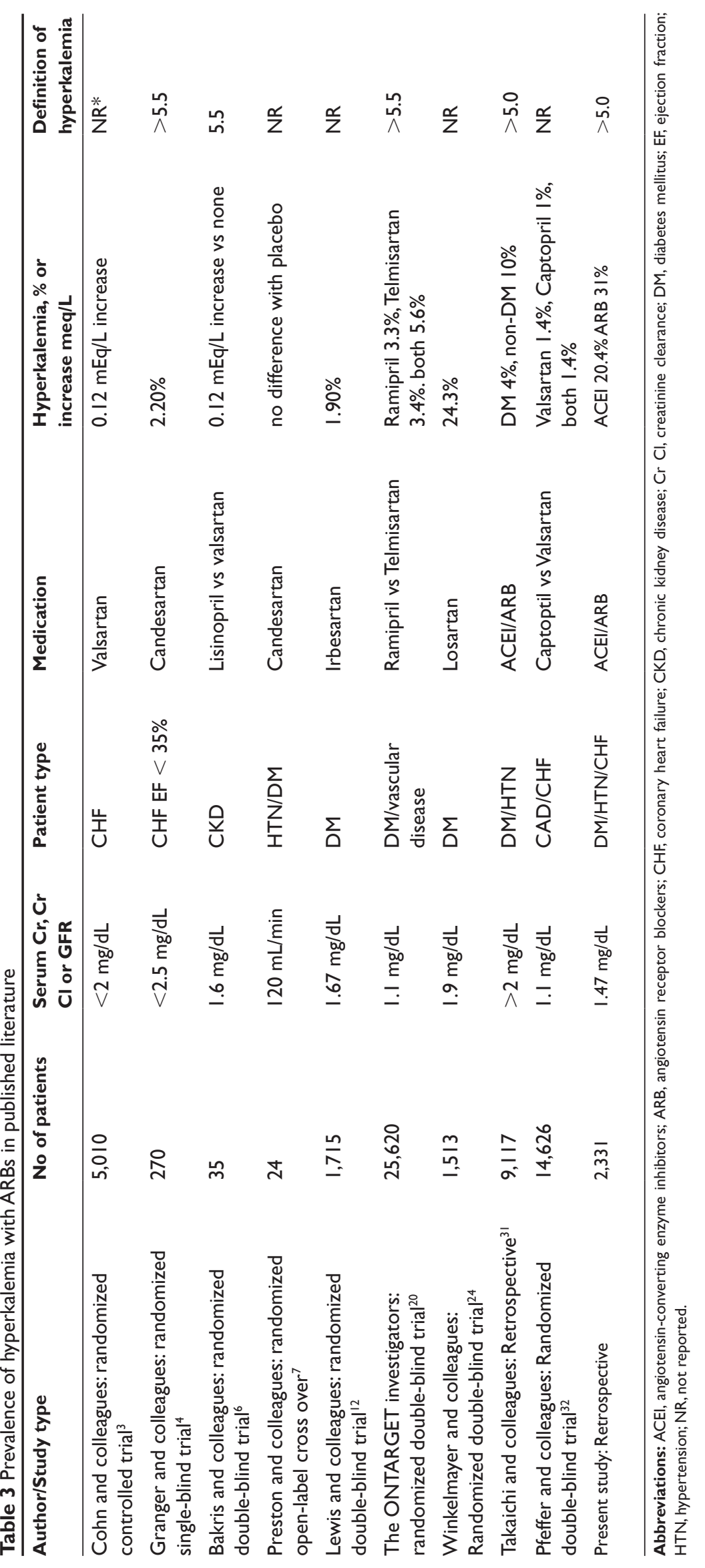




\section{Disclosure}

The authors report no conflicts of interest in this work.

\section{References}

1. Jessup M, Brozena S. Heart failure. N Engl J Med. 2003;348:2007-2018.

2. Massie BM, Armstrong PW, Cleland JG, et al. Toleration of high doses of angiotensin converting enzyme inhibitors in patients with chronic heart failure: Results from the Atlas trial. Arch Intern Med. 2001;161(2):165-171.

3. Cohn JN, Tognoni G; Valsartan Heart Failure Trial Investigators. Randomized trial of the angiotensin receptor blocker, valsartan, in chronic heart failure. N Engl J Med. 2001;345(23):1667-1675.

4. Granger CB, Ertl G, Kuch J, et al. Randomized trial of candesartan in the treatment of patients with congestive heart failure and a history of intolerance to angiotensin converting enzyme inhibitors. Am Heart $J$. 2000;139:609-617.

5. Blake J, Deverux RV. Differential effects of direct antagonism of AII receptor blockers compared to ACE inhibitors on serum potassium levels and azotemia in patients with severe congestive heart failure. Congestive Heart Fail. 2000;6:193-196.

6. Bakris G, Siomos M, Richardson D, et al. ACE inhibition or angiotensin receptor blockade: Impact on potassium in renal failure. Kidney Int. 2000;58:2084-2092.

7. Preston RA, Baltodano NM, Alonso AB, Epstein M. Comparative effects on dynamic renal potassium excretion of ACE inhibition versus angiotensin receptor blockade in hypertensive patients with type 2 diabetes mellitus. J Clin Pharmacol. 2002;42:754-761.

8. Packer M, Coats AJS, Fowler MB. Effect of carvedilol on survival in severe chronic heart failure. N Engl J Med. 2001;344:1651-1658.

9. Pitt B, Remm W, Zannad F, et al. The effect of spironolactone on morbidity and mortality in patients with severe heart failure. $N$ Engl $J$ Med. 1999;341:709-717.

10. Pitt B, Remm W, Zannad F, et al. Eplerenone post acute myocardial infarction heart failure efficacy and survival study Investigators. Eplerenone, a selective aldosterone blocker, in patients with left ventricular dysfunction after myocardial infarction. $N$ Engl $J$ Med. 2003;348:1309-1321.

11. Ahmed A. American College of Cardiology/American Heart Association chronic heart failure evaluation and management guidelines: Relevance to the geriatric practice. Am J Geriatr Soc. 2003;51:123-126.

12. Lewis EJ, Hunsicker LG, Clark WR, et al; For Collaborative Study Group. Renoprotective effect of angiotensin receptor antagonist irbesartan in patients with nephropathy due to type 2 diabetes. $N$ Engl $J$ Med. 2001;345:851-860.

13. Cadnapaphornchai MA, McFann K, Strain JD, Masoumi A, Schrier RW. Prospective change in renal volume and function in children with ADPKD. Clin J Am Soc Nephrol. 2009;4(4):820-829.

14. Nutahara K, Higashihara E, Horie S, et al. Calcium channel blocker versus angiotensin II receptor blocker in autosomal dominant polycystic kidney disease. Nephron Clin Pract. 2005;99(1):18-23.

15. Kanno Y, Okada H, Yamaji Y, Nakazato Y, Suzuki H. Angiotensin-converting-enzyme inhibitors slow renal decline in IgA nephropathy, independent of tubulointerstitial fibrosis at presentation. QJM. 2005;98(3):199-203.
16. Li PK, Leung CB, Chow KM; For HKVIN Study Group. Hong Kong study using valsartan in IgA nephropathy (HKVIN): a double-blind randomized, placebo-controlled study. Am J Kidney Dis. 2006; 47(5):751-760.

17. Taal M, Brenner B. Combination ACEI and ARB therapy: Additional benefit in renoprotection. Curr Opin Nephrol Hypertens. 2000;11:377-381

18. Segura J, Praga M, Rodicio JL, Ruilope LM. Combination is better than monotherapy with ACE inhibitor or angiotensin receptor antagonist at recommended doses. J Renin Angiotensin Aldosterone Syst. $2003 ; 4: 43-47$.

19. Dillon JL. Angiotensin converting enzyme inhibitors and angiotensin receptor blockers for IgA nephropathy. Semin Nephrol. 2004;24:218-224

20. The ONTARGET Investigators, Yusuf S, Teo KK, et al. Telmisartan, ramipril, or both in patients at high risk for vascular events. $N$ Engl $J$ Med. 2008;358:1547-1559.

21. Knoll GA, Saghagal A, Nair RC, Grhahm J, et al. Renin-angiotensin system blockade and the risk of hyperkalemia in chronic hemodialysis patients. Am J Med. 2002;112:110-114.

22. Fang J, Modhavan S, Cohen H, Alderman MH. Serum potassium and cardiovascular mortality. J Gen Intern Med. 2000;15:8850-890.

23. Charytan D, Goldfarb D. Indications for hospitalization of patients with hyperkalemia. Arch Intern Med. 2000;160:1605-1611.

24. Winkelmayer WC, Zhang Z, Shainfar S, et al. Efficacy and safety of angiotensin II receptor blockade in elederly patients with diabetes. Diabetes Care. 2006;29:2210-2217.

25. Appel GB, Radhkrishnan J, Avram M, et al. Analysis of metabolic parameters as predictors of risk in the RENAAL study. Diabetes Care. 2003;6:1402-1407.

26. Don BR, Sebastian A, Cheitlin M, Christiansen M, Schambelan M. Pseudohyperkalemia caused by fist clenching during phlebotomy. N Engl J Med. 1990;322:1290-1292.

27. Shlipak MG. Pharmacology of heart failure in patients with renal insufficiency. Ann Intern Med. 2003;138:917-924.

28. Heywood T, Fonarow G, Costanzo M, Mathur V, et al. High prevalence of renal dysfunction and its impact on outcome in 118465 patients hospitalized with acute decompensated heart failure: A report from the ADHERE data base. J Card Fail. 2007;13:422-430.

29. Juurlink DN, Mamdani MM, Lee DS, et al. Rates of hyperkalemia after publication of randomized aldactone evaluation study. $N$ Engl J Med. 2004;351:543-551.

30. Palmer BF. Current concepts: Managing hyperkalemia caused by inhibitors of the renin angiotensin aldosterone system. $N$ Engl J Med. 2004;351:543-551.

31. Takaichi K, Takemoto F, Ubara Y, Mori Y. Analysis of factors causing hyperkalemia. Intern Med. 2007;46:823-829.

32. Pfeffer MA, McMurray JJ, Velazquez EJ, et al. for the Valsartan in Acute Myocardial Infarction Trial Investigators: Valsartan, captopril, or both in myocardial infarction complicated by heart failure, left ventricular dysfunction, or both. $N$ Engl J Med. 2003;49:1893-1906.
Therapeutics and Clinical Risk Management

\section{Publish your work in this journal}

Therapeutics and Clinical Risk Management is an international, peerreviewed journal of clinical therapeutics and risk management, focusing on concise rapid reporting of clinical studies in all therapeutic areas, outcomes, safety, and programs for the effective, safe, and sustained use of medicines. This journal is indexed on PubMed Central, CAS,

\section{Dovepress}

EMBase, Scopus and the Elsevier Bibliographic databases. The manuscript management system is completely online and includes a very quick and fair peer-review system, which is all easy to use. Visit $\mathrm{http}: / /$ www.dovepress.com/testimonials.php to read real quotes from published authors. 